\title{
Borici-Creutz fermions on 2-dim lattice
}

\author{
Jishnu Goswami* \\ Physics Department, Indian Institute of Technology Kanpur, Kanpur-208016, India \\ E-mail: jgoswami@itk.ac.in
}

\section{Dipankar Chakrabarti}

Physics Department, Indian Institute of Technology Kanpur, Kanpur-208016, India

E-mail: dipankareittk.ac.in

\section{Subhasish Basak}

School of Physical Sciences, NISER, Jatni-752050, India

E-mail: sbasak@niser.ac.in

Minimally doubled fermion proposed by Creutz and Borici is a promising chiral fermion formulation on the lattice. In this work, we present excited state mass spectroscopy for the meson bound states in Gross-Neveu model using Borici-Creutz fermion. The lowest lying meson mass in 2-dimensional QED is also obtained using Borici-Creutz fermion. And the fermion mass dependence of lowest lying meson mass is also shown here.

34th International Symposium on Lattice Field Theory 24-30 July 2016

University of Southampton, UK

\footnotetext{
* Speaker.
} 


\section{Introduction}

Minimally doubled fermions in recent times have drawn attention as promising lattice formulations of chiral fermion. There are two minimally doubled fermion formulations, one is by Karsten[1] and Wilczek[2] and the other one was developed by Creutz[3] and Borici[4]. Both the formulations break the hypercubic symmetry on the lattice [5] and thus allow non-covariant counter terms. So, the important question is how bad the effects of the symmetry breaking are in a numerical simulation. It was shown that a consistent renormalizable theory for minimally doubled fermion can be constructed by fixing only three counter terms allowed by the symmetry and the counter terms for $\mathrm{BC}$ action at one loop in perturbation theory have been evaluated [6, 7]. But, till date, sufficient numerical studies of the minimally doubled fermions have not been done. The purpose of this work is to investigate Borici-Creutz(BC) formulation numerically in some simple models. It was shown that in presence of gauge background with integer-valued topological charge, $\mathrm{BC}$ action satisfies the Atiyah-Singer index theorem[8]. In [9], using BC fermion we have shown a chiral phase transition in the Gross-Neveu model. In this work, we evaluate the meson spectroscopy of the Gross-Neveu model as well as in 2D QED $\left(\mathrm{QED}_{2}\right)$ with $\mathrm{BC}$ fermion. Chiral and parity-broken(Aoki) phase structures of the Gross-Neveu model have been studied for Wilson and Karsten-Wilczek fermions [10, 11]. By hybrid Monte Carlo(HMC) simulation we investigate the excited state spectrum of the lattice Gross-Neveu model. Study of the excited state spectroscopy in the Gross-Neveu model with Wilson fermion [12] found the ground state as the only bound state and the other excited states as scattering states. With the BC fermion in this work, we have obtained three states, two of them are bound states (ground state and one excited state) and the third one appears to be a scattering state. Next we investigate the meson mass spectrum in QED in two dimension. $\mathrm{QED}_{2}$ having confinement serves as a toy model for $\mathrm{QCD}$ and hence $\mathrm{QED}_{2}$ or Schwinger model has been studied to great extent on lattice (see $[13,14]$ and references therein). Schwinger model using Hamiltonian formalism on lattice has been investigated in [15]. $\mathrm{QED}_{2}$ also serves as a good toy model for numerical study of chiral fermions. A 2-flavor Schwinger model with light fermions have been studied with dynamical overlap fermion [16, 17].

\section{Spectroscopy of the Gross-Neveu model}

The free $\mathrm{BC}$ action in $2 \mathrm{D}$ is written as,

$$
\begin{aligned}
S= & \sum_{n}\left[\frac{1}{2} \sum_{\mu} \bar{\psi}_{n} \gamma_{\mu}\left(\psi_{n+\mu}-\psi_{n-\mu}\right)-\frac{i r}{2} \sum_{\mu} \bar{\psi}_{n}\left(\Gamma-\gamma_{\mu}\right)\left(2 \psi_{n}-\psi_{n+\mu}-\psi_{n-\mu}\right)\right. \\
& \left.-i\left(2-c_{3}\right) \bar{\psi}_{n} \Gamma \psi_{n}+m_{0} \bar{\psi}_{n} \psi_{n}\right]
\end{aligned}
$$

where, $\mu=1,2$ and $\Gamma=\frac{1}{2}\left(\gamma_{1}+\gamma_{2}\right)$ satisfies $\left\{\Gamma, \gamma_{\mu}\right\}=1$. Including four-fermion interactions, the Gross-Neveu model on lattice is given by

$$
\begin{aligned}
S= & \sum_{n}\left[\frac{1}{2} \sum_{\mu} \bar{\psi}_{n} \gamma_{\mu}\left(\psi_{n+\mu}-\psi_{n-\mu}\right)-\frac{i r}{2} \sum_{\mu} \bar{\psi}_{n}\left(\Gamma-\gamma_{\mu}\right)\left(2 \psi_{n}-\psi_{n+\mu}-\psi_{n-\mu}\right)\right. \\
& -i\left(2-c_{3}\right) \bar{\psi}_{n} \Gamma \psi_{n}+m_{0} \bar{\psi}_{n} \psi_{n}-\frac{g^{2}}{2 N}\left[\left(\bar{\psi}_{n} \psi_{n}\right)^{2}+\left(\bar{\psi}_{n} i \Gamma \psi_{n}\right)^{2}\right]
\end{aligned}
$$


where, $g$ is the coupling constant which we consider the same for both four point (scalar and vector) interactions and we set $r=1$ in our calculations. Since the parity is broken by the BC action, a counter term $c_{3}$ is added to it. Detailed discussion about the $c_{3}$-term can be found in [9]. The action is rewritten explicitly in terms of the auxiliary fields as

$$
S=\sum_{m, n} \bar{\psi}_{m} M_{m n} \psi_{n}+\frac{N}{2 g^{2}}\left(\sigma^{2}+\pi_{\Gamma}^{2}\right)
$$

where $N$ is the number of flavors. The auxiliary fields are

$$
\sigma=-\frac{g^{2}}{N}(\bar{\psi} \psi), \quad \pi_{\Gamma}=-\frac{g^{2}}{N}(\bar{\psi} i \Gamma \psi) .
$$

For meson mass spectrum calculation, we need to evaluate the correlators

$$
C_{i j}(t)=\left\langle O_{i}(t) O_{j}^{\dagger}(0)\right\rangle .
$$

In absence of the orbital angular momentum in 2D, the interpolators $\left(O_{i}\right)$ are labelled by parity and charge conjugation only. We need to choose appropriate operators which have good overlaps with the low lying states. For the meson spectroscopy, we consider only the odd parity interpolators. The even parity interpolators correspond to condensate[12] and are not considered. Under parity $\psi(x, t) \rightarrow \gamma_{2} \psi(-x, t)$ and the odd parity interpolators can be constructed with $\gamma_{1}$ or $\gamma_{5}$. Along with the local source, one can also construct the interpolators with the fields at different lattice sites shifted along the spatial direction ie., with $\psi(x \pm n, t)$. If one considers a relative negative sign in between $\psi(x+n, t)$ and $\psi(x-n, t)$ then this corresponds to a derivative source which are found to be important for excited state spectroscopy $[12,18]$. Here we list some of the parity odd interpolators for the GN model which we expect to couple to ground state as well as excited states:

$$
\begin{aligned}
& O_{1}(t)=\bar{\psi}(x, t) \gamma_{5} \psi(x, t) \\
& O_{2}(t)=\frac{1}{4}\left((\bar{\psi}(x+m, t)-\bar{\psi}(x-m, t)) \gamma_{5}(\psi(x+n, t)-\psi(x-n, t)),(m=3, n=3)\right. \\
& O_{3}(t)=\frac{1}{4}\left((\bar{\psi}(x+m, t)-\bar{\psi}(x-m, t)) \gamma_{5}(\psi(x+n, t)-\psi(x-n, t)),(m=5, n=3)\right. \\
& O_{4}(t)=\frac{1}{4}\left((\bar{\psi}(x+m, t)-\bar{\psi}(x-m, t)) \gamma_{1}(\psi(x+n, t)-\psi(x-n, t)),(m=4, n=3)\right. \\
& O_{5}(t)=\frac{1}{4}\left((\bar{\psi}(x+m, t)+\bar{\psi}(x-m, t)) \gamma_{1}(\psi(x+n, t)-\psi(x-n, t)),(m=5, n=3),\right.
\end{aligned}
$$

where sum over $x$ is implied in order to have zero momentum states and $\gamma_{5}=i \gamma_{1} \gamma_{2}$. All the interpolators are odd under $C$-parity $(C=-1)$. With different values of $m$ and $n$, we can have different interpolators, they do not couple to new states but only reproduce the similar results. The effective masses are extracted from the correlators at different time slices by the formula

$$
M_{e f f}=\ln \left(\frac{c(t)}{c(t+1)}\right)
$$

The diagonal correlators $C_{i i}$ and corresponding effective masses are shown in Fig.1(a) and (b) for $m_{0}=0.03$ and $\beta=0.7$. The small value of mass is taken to be close to the massless limit. As shown in Fig. 1(b), except for the ground state, this procedure is not suitable to extract the excited states. 
(a)

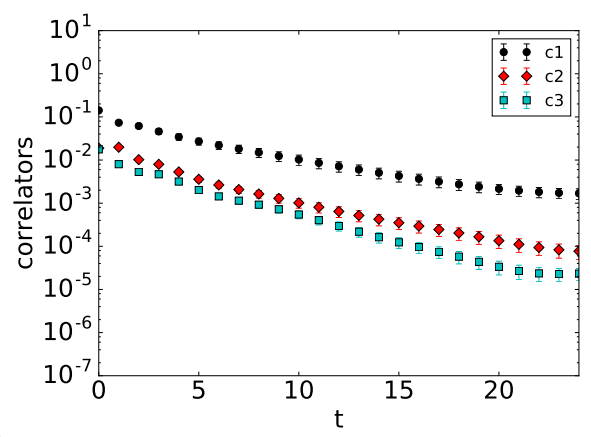

(b)

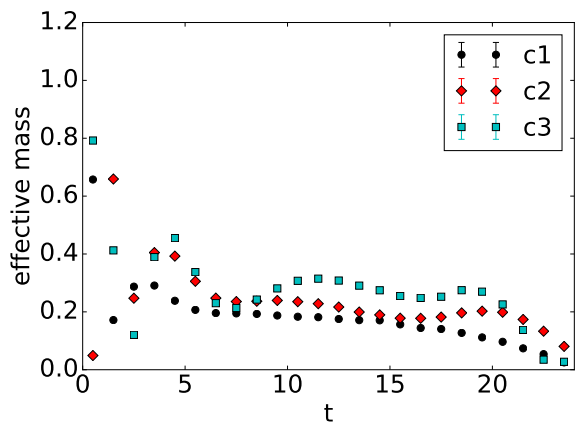

Figure 1: Diagonal correlators (in the plots $c 1 \equiv C_{11}$, etc.) and effective mass of meson in GN model for $16 \times 48$ lattice

(a)

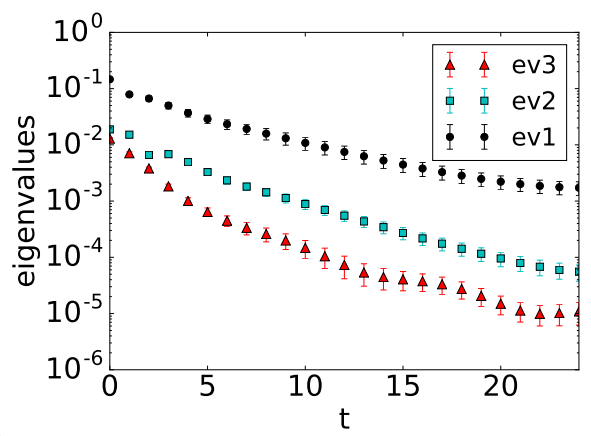

(c)
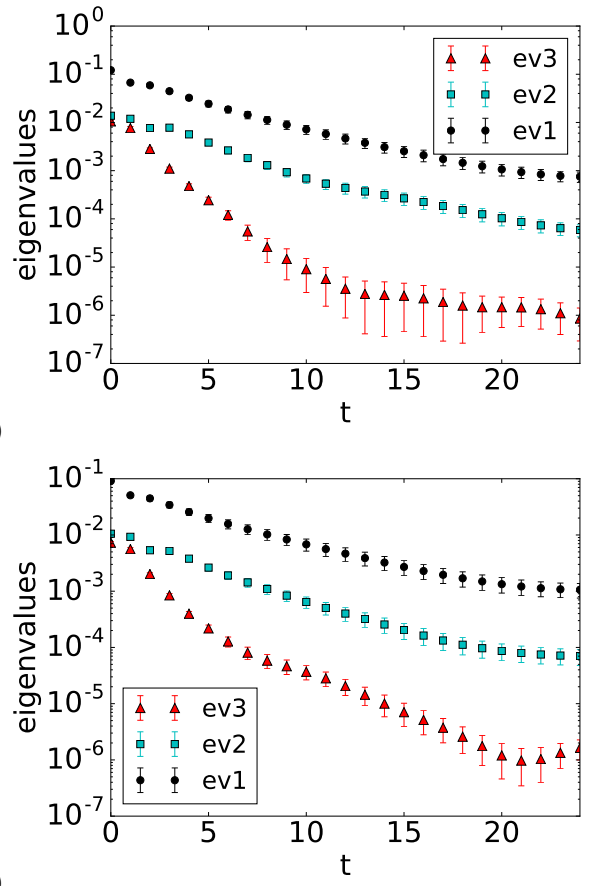

(e)

Figure 2: Eigenvalues and effective mass of the correlators. (a) and (b) for $16 \times 48$; (c) and (d) for $18 \times 48$; and (e) and (f) for $24 \times 48$ lattices.

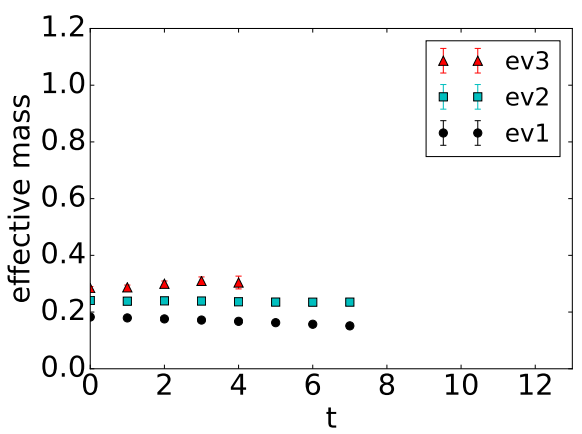

(b)

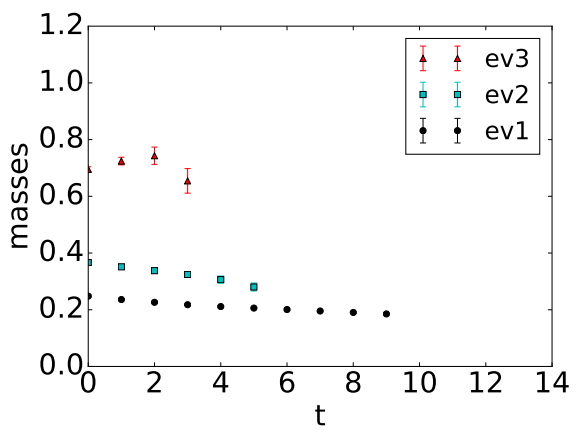

(d)

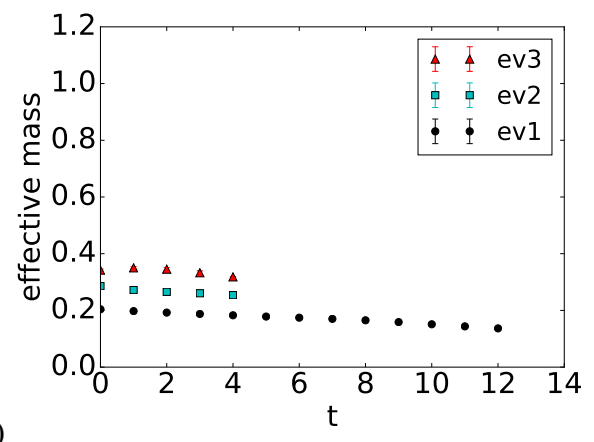

(f) 


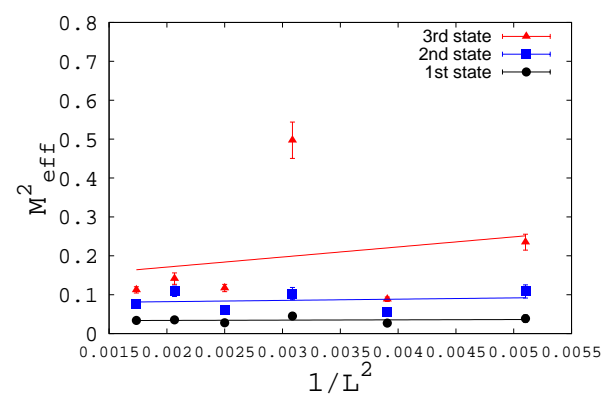

Figure 3: Volume dependence of the effective mass.

The variational method $[19,20]$ provides a better picture for the excited state meson spectroscopy. In this method, one solves the generalized eigenvalue problem

$$
C(t) \vec{v}^{(n)}=\lambda^{(n)}(t) C\left(t_{0}\right) \vec{v}^{(n)},
$$

where $C(t)$ is the $N \times N$ correlation matrix constructed from $N$ interpolators $O_{i},(i=1,2 \cdots, N)$. The $n$-th eigenvalue behaves as

$$
\lambda^{(n)}(t)=e^{-\left(t-t_{0}\right) E_{n}}\left[1+\mathscr{O}\left(e^{-\left(t-t_{0}\right) \Delta_{n}}\right)\right],
$$

where $E_{n}$ is the energy of the $n$-th state and $\Delta_{n}$ is the energy gap between the neighboring states. In Fig. 2 we show the eigenvalue and effective mass plots by solving the generalized eigen value problem for $16 \times 48,18 \times 48$, and $24 \times 48$ lattices with $O_{1}, O_{2}$ and $O_{3}$ interpolators. We are unable to get any extra stable mass values by increasing the matrix dimension of the correlator basis and the results become noisier with more correlators. So, we present the results with only three correlators. In Fig.3, the volume dependence of the effective masses are shown. The ground state and the first excited state show no volume dependence and hence can be considered as bound states. The second excited state however shows volume dependence. Specially, for $18 \times 48$ lattice size, we get an anomalously large mass for the second excited state. The fit for the second excited state shown in Fig. 3 includes this anomalous point. In general, scattering states show strong volume dependence and increase linearly with $1 / L^{2}$, the volume dependence of the second excited state in our case is not very conclusive. But looking at the fit of the points we expect it to be a scattering state. The results can be contrasted with [12], where except the ground state, all the excited states show strong volume dependence and are scattering states.

\section{Meson in 2D QED}

In this section, we extend the study of spectroscopy with $\mathrm{BC}$ fermion formulation to gauge theory. For this purpose, we implement the $\mathrm{BC}$ fermion in a $2 \mathrm{D} U(1)$ gauge theory and extract the meson masses. QED in 2D is also a confined theory and serves as a good toy model for QCD. The lattice action with $\mathrm{BC}$ fermion reads,

$$
S=\beta \sum_{p}\left[1-\frac{1}{2}\left(U_{p}+U_{p}^{\dagger}\right)\right]+\phi^{\dagger}\left(D^{\dagger} D\right)^{-1} \phi .
$$


where $U_{p}$ is the Wilson Plaquette action with

$$
U_{p}=U_{i, \mu} U_{i+\mu, v} U_{i+v, v}^{\dagger} U_{i, v}^{\dagger} .
$$

where, $i$ is the site index and $\mu, v$ are the directions and $D$ is the BC Dirac operator defined as

$$
\begin{aligned}
D_{m n}= & \frac{1}{2}\left(\gamma_{\mu}+i\left(\Gamma-\gamma_{\mu}\right)\right) U_{\mu}(n-\mu) \delta_{n, m+\mu}- \\
& \frac{1}{2}\left(\gamma_{\mu}-i\left(\Gamma-\gamma_{\mu}\right)\right) U_{\mu}^{\dagger}(n) \delta_{n, m-\mu}-\left(\left(2-c_{3}\right) i \Gamma-m_{0}\right) \delta_{m, n} .
\end{aligned}
$$

Here we consider only the lowest meson state. The correlator with operator $O_{1}(t)$ couples to the

(a)

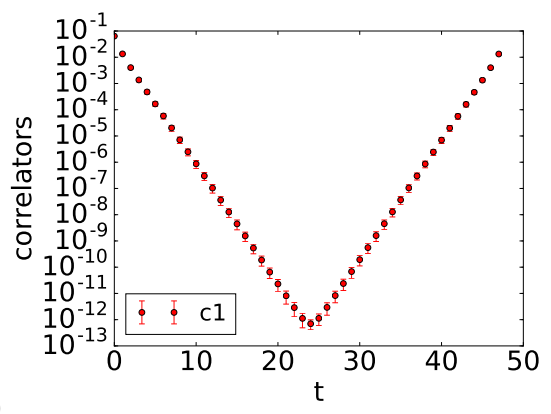

(b)

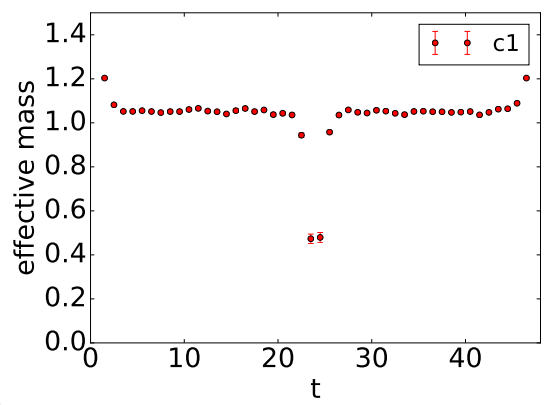

Figure 4: Effective mass of meson in 2D QED for $m_{0}=0.05$ and $\beta=0.3$.

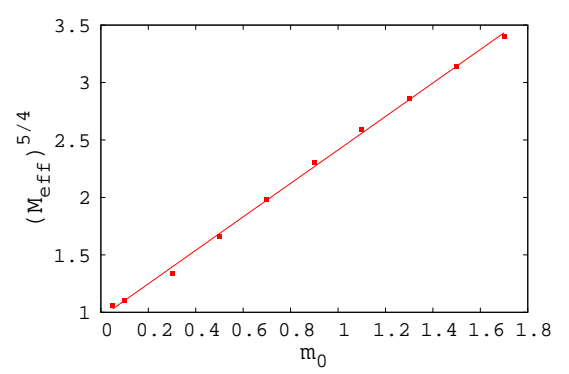

Figure 5: Fermion mass dependence of the effective pion mass in $\mathrm{QED}_{2}$ for a fixed $\beta=0.3$.

ground state and provides the mass for the lowest state. In Fig.4(a) we have shown the correlator at different time slices and the effective meson mass in 2D QED. The Schwinger model in continuum can be written as a bosonic theory. The pion mass in the bosonized theory can be exactly calculated[21,22] and for $m_{0} \ll g$, can be written as

$$
\begin{aligned}
M_{e f f} & =A m_{0}^{N_{f} /\left(N_{f}+1\right)} g^{1 /\left(N_{f}+1\right)}=A m_{0}^{5 / 4} g^{1 / 5} \quad\left(\text { for } \mathrm{N}_{\mathrm{f}}=4\right), \\
& =A m_{0}^{4 / 5} \beta^{-1 / 10}
\end{aligned}
$$

where $A$ is a constant and $\beta=1 / g^{2}$. In Fig.5, we show the fermion mass dependence of the effective pion mass $\left(M_{e f f}^{5 / 4} \propto m_{0}\right)$. The plot is done for small $\beta$ so that $m_{0}$ is always less than $g$. The lattice data are in well agreement with the analytic result. 


\section{Summary}

Minimally doubled fermions may provide an efficient lattice formalism to study chiral fermion which is expected to be computationally cheaper than the other existing lattice formalisms. Since, both the minimally doubled fermion formulations (KW and $\mathrm{BC}$ ) break hypercubic symmetries on the lattice, they require non-covariant counter terms. In this work, we have studied the BC fermion in some simple models. We have extracted the excited state mass spectrum in Gross-Neveu model using $\mathrm{BC}$ fermion. We have also evaluated the lowest lying meson mass in $\mathrm{QED}_{2}$. For light fermion, the meson is much heavier that $2 m_{0}$ and for heavy fermion the meson mass becomes less than $2 m_{0}$ as the renormalized fermion mass becomes much smaller than the bare mass at strong coupling. Our investigations suggest that $\mathrm{BC}$ fermion formalism might be a promising alternative to study the chiral fermions on a lattice.

J. G. likes to thank Stephan Dürr for his useful comments.

\section{References}

[1] L.H. Karsten, Phys. Lett. B 104,315 (1981).

[2] F. Wilczek, Phys. Rev. Lett. 59,2397 (1987).

[3] M. Creutz, JHEP 0804, 017(2008); Pos LAT2008, 080 (2008).

[4] A. Borici, Phys. rev. D. 78, 074504 (2008).

[5] P. F. Bedaque, M. I. Buchoff, B.C. Tiburzi, A. Walker-Loud, Phys. Lett. B. 662,449 (2008).

[6] S. Capitani, J. Weber, H. Wittig, Phys. Lett. B681, 105 (2009).

[7] S. Capitani, M. Creutz, J. Weber, H. Wittig, JHEP 1009, 027 (2010).

[8] D. Chakrabarti, S. J. Hands, A. Rago, JHEP 0906, 060 (2009).

[9] J. Goswami, D. Chakrabarti and S. Basak, Phys. Rev. D 91, no. 1, 014507 (2015).

[10] M. Creutz, T. Kimura, T. Misumi, Phys. Rev. D.83, 094506 (2011).

[11] T. Misumi, JHEP 1208, 068 (2012).

[12] J. Danzer and C. Gattringer, PoS LAT 2007, 092 (2007).

[13] C. Gutsfeld, H. A. Kastrup and K. Stergios, Nucl. Phys. B 560, 431 (1999).

[14] C. Gattringer et. al Phys. Lett. B 466,287 (1999).

[15] K. Cichy, A. Kujawa-Cichy and M. Szyniszewski, Comput. Phys. Commun. 184, 1666 (2013).

[16] L. Giusti, C. Hoelbling and C. Rebbi, Phys. Rev. D 64, 054501 (2001).

[17] W. Bietenholz, I. Hip, S. Shcheredin and J. Volkholz, Eur. Phys. J. C 72, 1938 (2012).

[18] C. Gattringer et. all, Phys. Rev. D 78, 034501 (2008).

[19] C. Michael, Nucl. Phys. B 259, 58 (1985).

[20] M. Luscher and U. Wolff, Nucl. Phys. B 339, 222 (1990).

[21] A. V. Smilga, Phys. Rev. D 55, 443 (1997).

[22] Stephan Durr, Phys. Rev. D 85114503 (2012). 